\title{
Correction to: A Case-Control Study of Environmental and Occupational Risks of Leptospirosis in Sri Lanka
}

Marie Hellung Schønning, ${ }^{1}$ Matthew David Phelps, ${ }^{1}$ Janith Warnasekara, ${ }^{2}$ Suneth B. Agampodi, ${ }^{2}$ and Peter Furu $\mathbb{1}^{1}$

${ }^{1}$ Global Health Section, Department of Public Health, University of Copenhagen, Oster Farimagsgade 5, 1014 Copenhagen K, Denmark

${ }^{2}$ Department of Community Medicine, Faculty of Medicine and Allied Sciences, Rajarata University of Sri Lanka, Saliyapura, Sri Lanka

Correction to: EcoHealth https://doi.org/10.1007/s10393-019-01448-w

The original version of this article unfortunately contained a mistake in one of the co-author's family name. The correct name should be Janith Warnasekara instead of Janith Warnasuriya. The original article has been corrected.

Published online: November 12, 2019

The original article can be found online at https://doi.org/10.1007/s10393-019-014 48-w.

Correspondence to: Peter Furu, e-mail: furu@sund.ku.dk 